\title{
Raman investigation of laser-induced structural defects of graphite oxide films
}

\author{
Valentino Romano ${ }^{1 *}$, Lorenzo Torrisi ${ }^{1}$, Mariapompea Cutroneo $^{2}$, Vladimir Havranek ${ }^{2}$ and Giovanna D'Angelo ${ }^{1 *}$ \\ ${ }^{1}$ Dipartimento di Science Matematiche ed Informatiche, Scienze Fisiche e Scienze della Terra, Università di Messina, Viale F. \\ Stagno d'Alcontres 31, S. Agata, 98166 Messina, Italy. \\ ${ }^{2}$ Nuclear Physics Institute, CAS, 25068 Rez, Czech Republic.
}

\begin{abstract}
Since the beginning of intensive studies on graphene and graphitic materials, Raman spectroscopy has always been used as a characterisation technique. This is due to two main reasons: the non-destructive nature of this experimental technique and its ability to distinguish between the plethora of existing carbon materials. One of the most challenging research activities concerns the production of graphene microcircuits. To address this issue, a possible strategy is to directly reduce and pattern graphite oxide (GO) film by laser irradiation. The objective of this study is to evaluate the laser irradiation-induced structural changes on thin GO films by using Micro-Raman spectroscopy. We used as a source a Nd:YAG laser $(1064 \mathrm{~nm})$ and different laser fluences: $15 \mathrm{~J} / \mathrm{cm}^{2}, 7.5 \mathrm{~J} / \mathrm{cm}^{2}$ and $5 \mathrm{~J} / \mathrm{cm}^{2}$. We have analyzed the modifications of the main Raman contributions of these graphitic materials: the $D$ band (defect induced band), the $G$ band (band due to $\mathrm{sp}^{2}$ hybridized carbon atoms) and the $2 D$ band (D band overtone). In particular, we found out that our figure of merit (FOM) parameters, i.e. the intensity ratio $I_{D} / I_{G}$ (for the $D$ band and $G$ band) and $I_{2 D} / I_{G}$ (for the $2 D$ band and $G$ band), change with the laser fluences, revealing a different effect induced by the laser irradiation. The best results are found in the sample irradiated with $5 \mathrm{~J} / \mathrm{cm}^{2}$, suggesting that higher fluences do not lead to better results.
\end{abstract}

\section{Introduction}

Raman spectroscopy is a vibrational technique that is extremely sensitive to geometric structure and bonding within molecules. This sensitivity is useful for the study of the various allotropes of carbon (i.e. diamond, carbon nanotubes [1], buckminster fullerenes [2], graphene nanoribbons [3], etc.) where the different forms differ only in the relative position of their carbon atoms and the nature of the chemical bonding. It is well known that graphene is characterised by properties of great potential: breaking strength $(\approx 40 \mathrm{~N} / \mathrm{m})$, room temperature thermal conductivity $\left(\approx 5000 \mathrm{~W} \mathrm{~m}^{-1} \mathrm{~K}^{-1}\right)$, Young modulus $(\approx 1$ $\mathrm{TPa})$ and so on [4]. Furthermore the electron mobility of graphene $\left(1-2 \times 10^{5} \mathrm{~cm}^{2} / \mathrm{Vs}\right)$ is a hundred times higher than that of silicon [5], so graphene is a material of great potential for use in micro-electronics [6, 7]. However the manufacture of integrated circuits needs a transfer printing method on small size of graphene films [8]. Therefore, the quest for a suitable production method arised. One possibility is to reduce graphite oxide (GO). This material is obtained by oxidizing graphite by means of strong oxidizing compounds (like $\mathrm{KMnO}_{4}$ ) [9]. This procedure induces profound changes in the structure of graphite because epoxy and hydroxyl groups occur in the basal plane, while carboxylic groups arise at the edges $[10,11]$. These defects of the graphitic structure are responsible for the insulating or semiconducting properties of GO. The reduction of graphite oxide can be accomplished by means of several methods like dehydration, thermal reduction and UV-assisted photocatalysis [12]. Another option is to locally reduce and pattern graphite oxides (GO) by using laser ablation [13]. In fact high intensity laser beams (of the order of $10^{10} \mathrm{~W} / \mathrm{cm}^{2}$ ) induce change in the composition (due to hydrogen, oxygen and other impurities vaporisation), foil thickness, mechanical properties and morphology [14]. These are due to the high electron density and the high thermal conductivity of the carbon layers. In this context, it is also very important to understand and quantify the consequences of disorder induced by pulsed laser technologies on the electronic profile of GO. To evaluate the role of disorder, defects can be introduced in graphite by ion or laser irradiation [15]. In this work we present the results of a Micro - Raman spectroscopy investigation on GO samples irradiated by laser pulses with different fluences.

\section{The Raman spectra of pristine and defected graphene}

The Raman spectrum of pristine graphene is characterised by two main contributions: the $G$ band $\left(1582 \mathrm{~cm}^{-1}\right)$ and

\footnotetext{
*Corresponding authors: gdangelo@unime.it, vromano@unime.it
} 
the $2 D$ band (around $2700 \mathrm{~cm}^{-1}$ ) [16]. (Fig. 1, top, shows an example of this spectrum, taken from [18]).

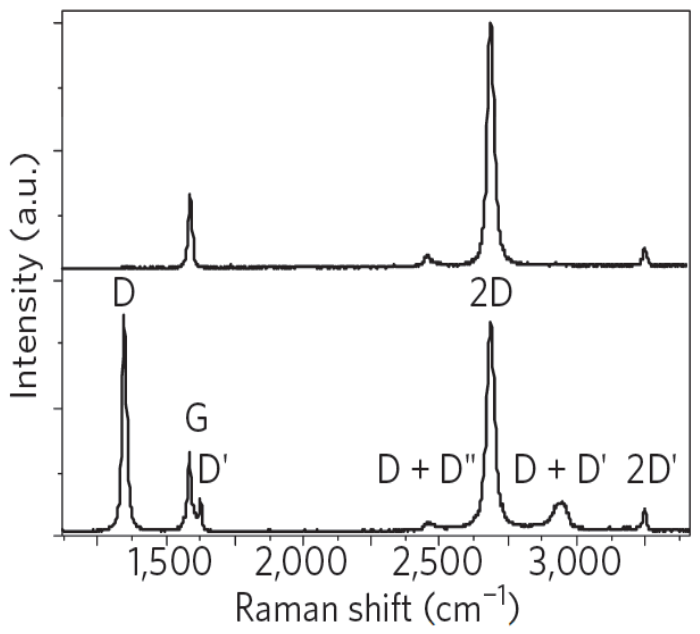

Fig. 1. Raman spectra of pristine (top) and defected (down) graphene [18].

The $G$ peak corresponds to the $E_{2 g}$ phonon at the Brillouin zone center. The $D$ peak is due to the breathing modes of $s p_{2}$ rings and requires a defect for its activation by double resonance. The $2 D$ peak is the second order of the $D$ peak. This is a single peak in monolayer graphene, whereas it splits in multi-layer graphene, reflecting the evolution of the band structure. The $2 D$ peak is always seen, even when no $D$ peak is present, since no defects are required for the activation of two phonons with the same momentum, one backscattered from the other [17]. In defected graphene and graphitic materials many other contributions occur at: $1350 \mathrm{~cm}^{-1}$ ( $D$ band), $1620 \mathrm{~cm}^{-1}\left(D^{\prime}\right.$ band), $2445 \mathrm{~cm}^{-1}$ ( $D+D^{\prime \prime}$ band), $2925 \mathrm{~cm}^{-1}$ ( $D+D^{\prime}$ 'band) and $3160 \mathrm{~cm}^{-1}$ (2D' band) [17]. A thorough analysis of the Raman spectra provides many information about the structure in graphene samples. For example the Raman spectrum of pristine graphene shows a single $G$ peak and a sharp $2 D$ peak, roughly four times more intense than the $G$ peak. It was observed that in few layers graphene the $2 D$ region is characterised by a multi-band (centered at about $2700 \mathrm{~cm}^{-1}$ ) and the insurgence of the medium frequency defect-induced contributions [18]. Thus, the intensity ratio for the $D, G$ and $2 D$ Raman bands is often used as a criterion to assess the graphene quality. Minimum of $I_{D} / I_{G}$ and maximum of $I_{2 D} / I_{G}$ are the indicators of the highest quality of graphene and, in the study case, provide information on the best conditions for the GO-to-graphene reduction. In fact, the first ratio characterizes the in-plane crystallite sizes [17], while $I_{2 D} / I_{G}$ is used to distinguish a single layer, from bilayer from few (less than 5) layers [18].

\section{Experimental}

\subsection{Material synthesis}

The graphite oxide (GO) foil was prepared by graphite oxidation adopting the permanganate oxidation method. Graphite ( $99.995 \%$ and $2-15 \mu \mathrm{m}$ ) was purchased by Alfa
Aesar and mixed with $\mathrm{H}_{2} \mathrm{SO}_{4}(96 \mathrm{wt} \%)$ and $\mathrm{H}_{3} \mathrm{PO}_{4}(85$ wt\%). After that, $\mathrm{KMnO}_{4}$ was added and the reaction mixture was heated to $50^{\circ} \mathrm{C}$ for 12 hours. Afterwards, the reaction mixture was quenched in ice (400 g) with hydrogen peroxide $(30 \mathrm{wt} \%)$, and the formed graphite oxide was separated by centrifugation. Nucleopore 0.45 $\mu \mathrm{m}$ was used to obtain GO foil by suction filtration of graphite oxide suspension [19].

\subsection{Laser treatment}

A Nd:YAG laser operating at fundamental frequency (1064 nm), $3 \mathrm{~ns}$ pulse duration, $300 \mathrm{~mJ}$ maximum pulse energy, $1 \mathrm{~mm}^{2}$ spot size focalization, about $10^{10} \mathrm{~W} / \mathrm{cm}^{2}$ intensity, operating in single pulse or up to $10 \mathrm{~Hz}$ repetition rate was employed in our investigations. Laser was employed to irradiate GO thin foils placed in the sample chamber using window and an external lens with $50 \mathrm{~cm}$ focal length for the light focalization. In order to reduce the ablation effect producing perforation at higher energies, the laser pulse energy was reduced to $150 \mathrm{~mJ}$, $75 \mathrm{~mJ}$ and $50 \mathrm{~mJ}$, employed with a fluence of $15 \mathrm{~J} / \mathrm{cm}^{2}$, $7.5 \mathrm{~J} / \mathrm{cm}^{2}$ and $5 \mathrm{~J} / \mathrm{cm}^{2}$, respectively.

The laser irradiation was monitored in-situ using a Joulemeter for the laser pulse energy control, an ion collector (IC) connected in time-of-flight configuration to a fast oscilloscope and a mass quadrupole spectrometer (MQS) for the gas degassing diagnostics [20]. The last two techniques have detected the presence of carbon and oxygen ions emitted from the laser ablation process. A scheme of the experimental set up is reported in Fig. 2.

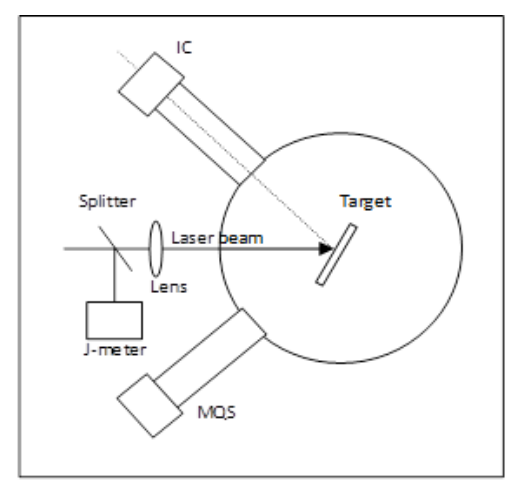

Fig. 2. Experimental set-up for graphite oxide irradiation.

\subsection{Characterisation}

Raman microscopy was used to investigate the structural transformations in GO samples. Raman measurements were performed with $532 \mathrm{~nm}$ excitation light by using the LabRam HR800 (Horiba Jobin Yvon) spectrometer with the $1800 \mathrm{gr} / \mathrm{mm}$ grating and a CCD camera cooled with liquid nitrogen. The laser power used was $1 \mathrm{~mW}$ and the spectra were taken with the $50 \mathrm{x}$ objective lens. The overall integration time was $100 \mathrm{~s}$. 


\section{Results and discussion}

The Tandetron $4130 \mathrm{MC}$ at the Tandetron laboratory of the Nuclear Physics Institute (Czech Republic) is compact, multipurpose linear electrostatic tandem accelerator system produced by High Voltage Engineering Europa (HVEE). The beam passes through an analysing magnet (providing mass and charge state of incoming ions) and ions are driven towards the beam line where the RBS-ERDA systems are located.

Rutherford Backscattering Spectrometry (RBS) and Elastic Recoil Detection Analysis (ERDA) were employed to evaluate the composition of GO foil. A probe of $\mathrm{He}^{+}$ions with energy of $1.9 \mathrm{MeV}$ and incident angle of $0^{\circ}$ with respect to the foil surface normal, was used for the measurements. During RBS analysis, an Ultra-Ortec PIPS detector detected $\mathrm{He}^{+}$ions backscattered at a laboratory scattering angle of $160^{\circ}$. In ERDA spectrum, hydrogen atoms that recoiled at a scattering angle of $35^{\circ}$ were registered using a detector covered by a $4 \mu \mathrm{m}$ Polypropylene foil to avoid the detection of the primary ions scattering. The ion current was $5.4 \mathrm{nA}$. RBS and ERDA spectra were evaluated using SIMNRA code [21]. During RBS and ERD analyses, several spectra are collected on different beam spots to avoid degradation effects of the sample and the final spectrum is obtained as the sum of the single collected spectra. The elemental compositions of GO obtained by RBS and ERD analyses are shown in Fig. 3 and presented in Tab. I.

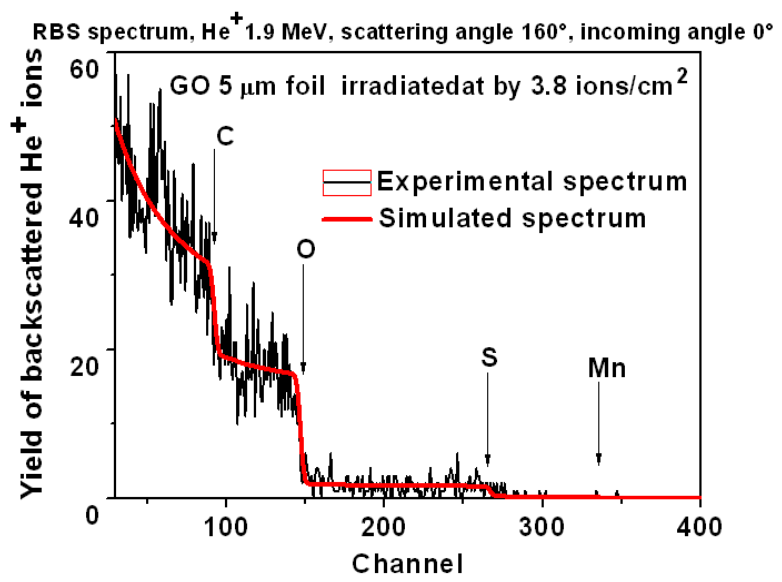

Fig. 3. Spectrum related GO foil obtained by Rutherford backscattering spectrometry.

Tab. I Elemental compositions of GO foils.

\begin{tabular}{|c|r|r|r|r|r|}
\hline \multirow{2}{*}{$\begin{array}{c}\text { Target/ } \\
\text { Thickness }\end{array}$} & \multicolumn{5}{|c|}{ Foils composition (at \%) } \\
\cline { 2 - 6 } & H & C & O & S & Mn \\
\hline GO $5 \mu \mathrm{m}$ & 18.4 & 47.6 & 33.1 & 0.75 & 0.046 \\
\hline
\end{tabular}

In this study we irradiated the GO samples using different laser fluences $\left(5 \mathrm{~J} / \mathrm{cm}^{2}, 7.5 \mathrm{~J} / \mathrm{cm}^{2}\right.$ and $\left.15 \mathrm{~J} / \mathrm{cm}^{2}\right)$. The irradiation procedure was performed in air atmosphere. During laser irradiation IC and MQS have monitored a high desorption of oxygen gas from the GO target, together emission of carbon and hydrogen, such as ions and neutral atoms. The ablated samples were investigated by means of Raman microscopy, the related spectra are shown and compared with the reference spectrum in Fig. 4.

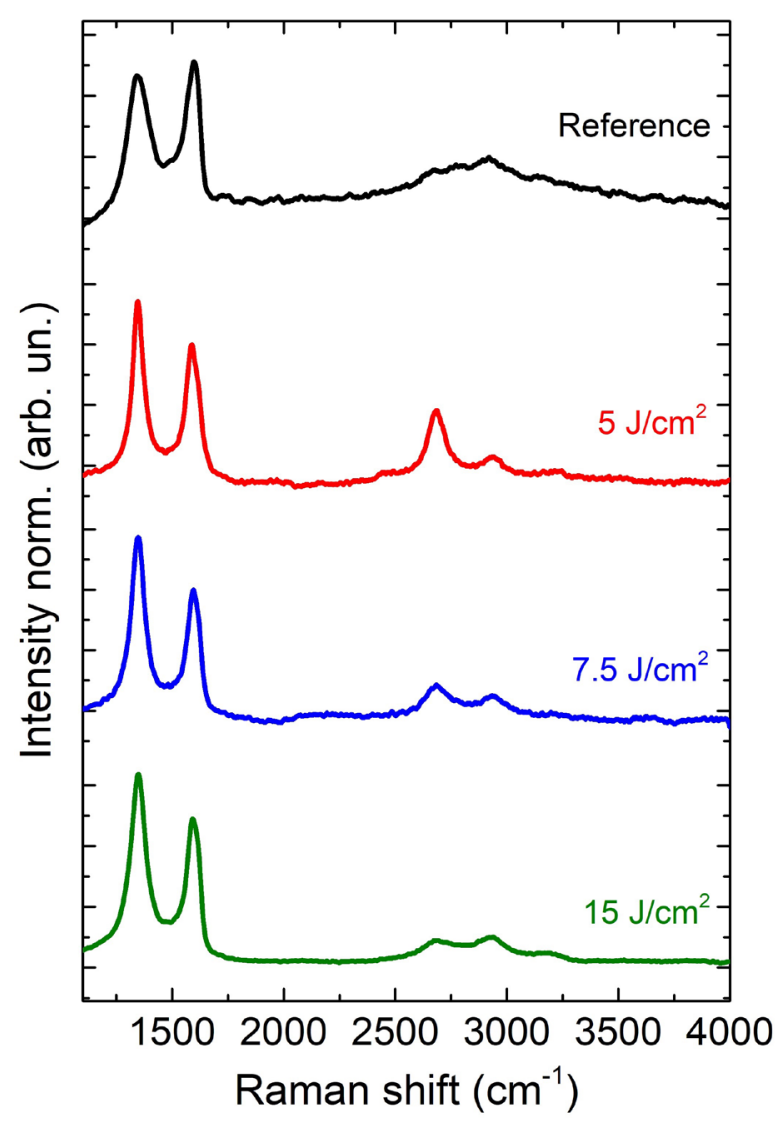

Fig. 4. Raman spectra of GO reference sample (black line), 5 $\mathrm{J} / \mathrm{cm}^{2}$ irradiated area (red line), $7.5 \mathrm{~J} / \mathrm{cm}^{2}$ irradiated area (blue line), $15 \mathrm{~J} / \mathrm{cm}^{2}$ irradiated area (green line). The spectra are normalized with respect to the $G$ peak intensity.

These spectra can be separated in two parts: the mediumRaman-shift region (below $2000 \mathrm{~cm}^{-1}$ ) and the highRaman-shift region (above $2000 \mathrm{~cm}^{-1}$ ). The first one is characterised by the $D$ and $G$ peaks, while the high Raman shift region is characterised by a broad bump, centred around the $2 D$ peak position, which is due to the appearance of the defect-induced contributions discussed in one of the previous sections. As concerns the reference spectrum, the $D$ and $G$ peaks have similar intensities while the bump around $2700 \mathrm{~cm}^{-1}$ has a low intensity. This can mean that the sample is made up by several graphene layers and that defects (e. g. structural, chemical, etc) are present. The spectra in the irradiated samples have a $D$ intensity which is higher than the $G$ one, which means that a more defects are present, although the $2 D$ peak is more intense than in the reference case.

To make these observations more quantitative, we used the intensity ratios $I_{D} / I_{G}$ and $I_{2 D} / I_{G}$ as figures of merit (FOM) of our study. Minimum of $I_{D} / I_{G}$ and maximum of $I_{2 D} / I_{G}$ underline that the ablation procedure leads to a better quality material. The values of the intensity ratios computed for the spectra of Fig. 4 are reported in Tab. II. 
Tab. II Computed values of the intensity ratios $I_{D} / I_{G}$ and $I_{2 D} / I_{G}$ for the Raman spectra shown in Fig. 4.

\begin{tabular}{|l|l|l|}
\hline \multicolumn{1}{|c|}{ Sample } & \multicolumn{1}{|c|}{$I_{D} / I_{G}$} & \multicolumn{1}{c|}{$I_{2 D} / I_{G}$} \\
\hline Reference & 0.96 & 0.68 \\
\hline $5 \mathrm{~J} / \mathrm{cm}^{2}$ & 1.14 & 0.78 \\
\hline $7.5 \mathrm{~J} / \mathrm{cm}^{2}$ & 1.17 & 0.69 \\
\hline $15 \mathrm{~J} / \mathrm{cm}^{2}$ & 1.17 & 0.69 \\
\hline
\end{tabular}

These values confirm that the irradiated GO samples are characterized by a higher number of defects, with respect to the reference sample, but they still are made up by a lower number of layers. We believe that there are two possible reasons for this observation. On the one hand, since the ablation procedure was carried out in air atmosphere, further oxide species can be created $(\mathrm{CO}$, $\mathrm{CO}_{2}$ ) inducing more structural and chemical defects [16]. On the other hand the irradiation causes the removal of several layers (so the $I_{2 D} / I_{G}$ ratio increases) but more edge defects are produced. These effects cause the formation of smaller crystalline regions, because edge defects and chemically different species limit the dimensions of the crystallites. The Raman results can be used to get some insight in this problem. In fact Tuinstra and Koenig demonstrated that the intensity ratio $I_{D} / I_{G}$ is inversely proportional to the in plane crystallites size and proposed a formula which is valid for the $514.5 \mathrm{~nm}$ excitation wavelength only $[22,23]$. A general equation, that can be used for every wavelength, is given in [17]:

$$
L_{a}(n m)=\left(2.4 \times 10^{-10}\right) \lambda_{\text {laser }}^{4}\left(I_{D} / I_{G}\right)^{-1}
$$

where $L_{a}$ denotes the crystallite size and $\lambda_{\text {laser }}$ the laser excitation wavelength. In Fig. 5 we report the values that we computed on the basis of our Raman spectra.

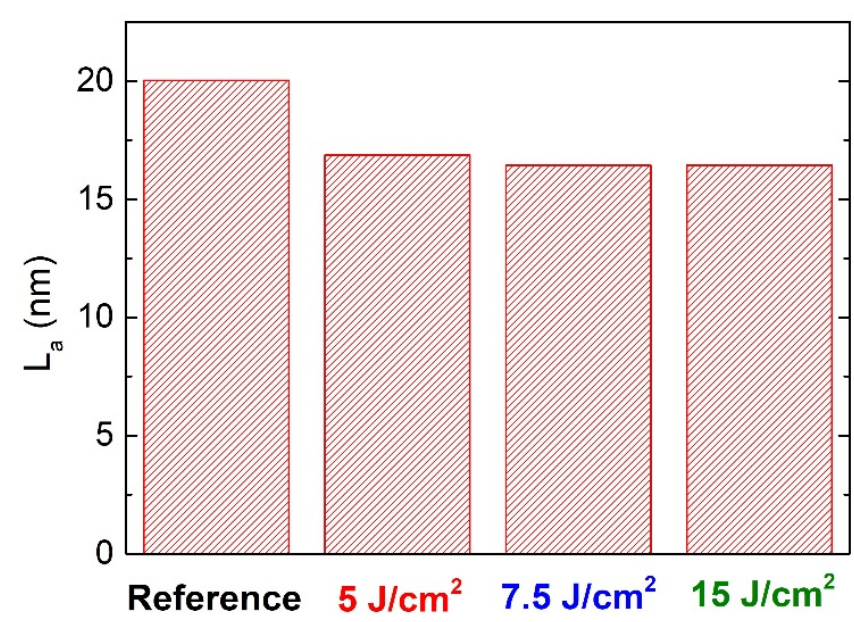

Fig. 5. Computed values of the crystallite size $L_{a}$ in nanometers.
These results confirm our hypothesis: the higher the laser fluence (i.e. the damage) to the GO sample is, the smaller the crystallites size become.

\section{Conclusions and perspectives}

To the best of our knowledge, this is the first time that Raman analysis of nano-second laser ablation of graphite oxide is investigated. Up to know only pico and femtosecond laser pulse sources have been used. Here we show that some improvements are still observed in this time domain. The best results concern the region irradiated with a $5 \mathrm{~J} / \mathrm{cm}^{2}$ laser fluence. However the ablation procedure leads to both a greater $I_{2 D} / I_{G}$ intensity ratio and an increase in the $I_{D} / I_{G}$ intensity ratio. We believe that this is due to the formation of carbon-oxide species, since the laser irradiation has been performed in air atmosphere. These results lead us to the idea of performing the same kind of study in inert atmosphere (trying to optimise this procedure). In this way we could be able to demonstrate if nano-second laser ablation can compete with pico and femto-second sources.

This work was supported by the "Research and Mobility" project of Messina University No. 74893496, scientifically coordinated by Professor L. Torrisi. The research has been also carried out at CANAM infrastructure LM2015056 and Czech Science Foundation (GACR No. 16-05167S).

\section{References}

1. M. S. Dresselhaus, G. Dresselhaus, R. Saito, A. Jorio, Phys. Rep. 409, 47-49 (2005).

2. D. S. Bethune, G. Meijer, W. C. Tang, H. J. Rosen, W. G. Golden, H. Seki, C. A. brown, M. S. de Vries, Chem. Phys. Lett. 179, 181-186 (1991).

3. L. Xie, H. Wang, C. Jin, X. Wang, L. Jiao, K. Suenaga, H. Dai, J. Am. Chem. Soc. 133, 10394-10397 (2011).

4. A. K. Geim, Science 324, 1530-1534 (2009).

5. C. Jacoboni, C. Canali, G. Ottaviani, A. A. Quaranta, Sol.St. Elect., 20, 77-89 (1977).

6. H. B. Heersche, P. Jarillo-Herrero, J. B. Oostinga, L. M. K. Vandersypen, A. F. Morpurgo, Nat. 446, 56-59 (2007).

7. G. Eda, M. Chhowalla, Nano Lett. 9, 814-818 (2009).

8. M. J. Allen, V. C. Tung, L. Gomez, Z. Xu, L. M. Chen, K. S. Nelson, C. W. Zhou, R. B. Kaner, Y. Yang, Adv. Mater. 21, 2098-2102 (2009).

9. J. Chen, B. Yao, C. Li, G. Shi, Carbon 64, 225-229 (2013).

10. G. Eda, M. Chhowalla, Adv. Mater. 22, $2392-2415$ (2010).

11. D. R. Dreyer, S. Park, C. W. Bielawski , R. S. Ruoff, Chem. Soc. Rev. 39, 228-240 (2010).

12. F. Bonaccorso, A. Lombardo, T. Hasan, Z. Sun, L. Colombo, A. C. Ferrari, Materials Today 15, 564-589 (2012).

13. Y. Zhang, L. Guo, S. Wei, Y. He, H. Xia, Q. Chen, H. Sun, F. Xiao, Nano Today 5, 15-20 (2010).

14. P. Kumar, K. S. Subrahmanyam, and C. N. R. Rao, Int. J. Nanosci. 10, 559-566 (2011). 
15. L. Tapaszto, G. Dobrik, P. Nemes-Incze, G. Vertesy, P. Lambin, L. P. Biro, Phys. Rev. B 78, 23 (2008).

16. R. Trusovas, K. Ratautas, G. Račiukaitis, J. Barkauskas, I. Stankevičienè, G. Niaura, \& R. Mažeikienè, Carbon 7, 574-582 (2012).

17. M. A. Pimenta, G. Dresselhaus, M. S. Dresselhaus, L. G. Cancado, A. Jorio, R. Saito, Phys. Chem. Chem. Phys. 9, 1276-1290 (2007).

18. A. C. Ferrari, D. M. Basko, Nat. Nano. 8, 235-246 (2013).

19. Ch. K. Chua and M. Pumera, Small 11, 1266-1272 (2015).

20. L. Torrisi, S. Gammino, A. M. Mezzasalma, A. M. Visco, J. Badziak, P. Parys, J. Wolowski, E. Woryna, J. Krasa, L. Laska, M. pfeifer, K. Rohlena and F.P. Boody, Appl. Surf. Sci. 227, 164-174 (2004).

21. M. Mayer, SIMNRA version 6.06, Max-Planck-Institut furlPlasmaphysik Garching, Germany, 2006, available at: http://home.mpcdf.mpg.de/ mam/].

22. F. Tuinstra, J. L. Koenig, J. Chem. Phys. 53, 3 (1970).

23. F. Tuinstra, J. L. Koenig, J. Compos. Mater. 4, 4 (1970). 